\title{
Article \\ Expression Level of Mature miR172 in Wild Type and StSUT4-Silenced Plants of Solanum tuberosum Is Sucrose-Dependent
}

\author{
Varsha Garg, Aleksandra Hackel and Christina Kühn *iD
}

Citation: Garg, V.; Hackel, A.; Kühn, C. Expression Level of Mature miR172 in Wild Type and StSUT4Silenced Plants of Solanum tuberosum Is Sucrose-Dependent. Int. J. Mol. Sci. 2021, 22, 1455. https://doi.org/ $10.3390 /$ ijms 22031455

Academic Editors: Iwona Morkunas, Philippe Jeandet, Mateusz Labudda and Magda Formela-Luboińska Received: 18 December 2020

Accepted: 27 January 2021

Published: 1 February 2021

Publisher's Note: MDPI stays neutral with regard to jurisdictional claims in published maps and institutional affiliations.

Copyright: (c) 2021 by the authors. Licensee MDPI, Basel, Switzerland. This article is an open access article distributed under the terms and conditions of the Creative Commons Attribution (CC BY) license (https:/ / creativecommons.org/licenses/by/ $4.0 /)$.
Department of Plant Physiology, Institute of Biology, Humboldt-Universität zu Berlin, Philippstr. 13, Building 12, 10115 Berlin, Germany; varsha.garg.1@hu-berlin.de (V.G.); hackelal@hu-berlin.de (A.H.)

* Correspondence: christina.kuehn@hu-berlin.de; Tel.: +49-30-2093-98342

\begin{abstract}
In potato plants, the phloem-mobile miR172 is involved in the sugar-dependent transmission of flower and tuber inducing signal transduction pathways and a clear link between solute transport and the induction of flowering and tuberization was demonstrated. The sucrose transporter StSUT4 seems to play an important role in the photoperiod-dependent triggering of both developmental processes, flowering and tuberization, and the phenotype of StSUT4-inhibited potato plants is reminiscent to miR172 overexpressing plants. The first aim of this study was the determination of the level of miR172 in sink and source leaves of StSUT4-silenced as well as StSUT4-overexpressing plants in comparison to Solanum tuberosum ssp. Andigena wild type plants. The second aim was to investigate the effect of sugars on the level of miRNA172 in whole cut leaves, as well as in whole in vitro plantlets that were supplemented with exogenous sugars. Experiments clearly show a sucrose-dependent induction of the level of mature miR172 in short time as well as long time experiments. A sucrose-dependent accumulation of miR172 was also measured in mature leaves of StSUT4-silenced plants where sucrose export is delayed and sucrose accumulates at the end of the light period.
\end{abstract}

Keywords: photoperiodic control; flowering; tuberization; miR172; sucrose transporter; sucrose signaling

\section{Introduction}

Sugar accumulation and starch biosynthesis in mature leaves follow day time dependent oscillations. Whereas the accumulation of transitory starch in leaves is strongly light-dark-dependent since the rate-limiting enzyme in starch biosynthesis, the ADPglucose pyrophosphorylase (AGPase) is regulated by light, the accumulation of soluble sugars in mature leaves oscillates during the day with maximum levels in the middle of the light period [1]. In accordance with this sugar accumulation the transcript amount of sucrose transporters follow a circadian rhythm and continue oscillation even under constant light conditions [1]. Whereas StSUT1, the main phloem loader in potato, show maximum levels $6 \mathrm{~h}$ after light set on (and circadian evening elements, EE) were found in the promoter region of this gene according to Harmer and Kay [2]. In turn, the StSUT2 gene show maximum levels in the early morning (and circadian morning elements (ME) were found in the cis-regulatory upstream region of the gene).

Sucrose transporter StSUT4 seems to be involved in sugar-dependent signaling processes and StSUT4-inhibited potato plants revealed a pleiotropic phenotype regarding carbon translocation and accumulation. Potato plants with down-regulated StSUT4expression are early flowering and develop tubers even under non-inductive long day (LD) conditions [1]. They show reduced internode elongation and produced reduced amounts of ethylene [3]. StSUT4-RNAi plants are far-red insensitive and do not show shade avoidance response if shaded by neighboring plants or if grown under far red enrichment. Sucrose 
efflux from mature leaves is significantly increased at the end of the light period and increased export of photo-assimilates from leaves is accompanied by increased amount of soluble sugars or starch in sinks such as developing tubers [1,3]. Increased sucrose export is not necessarily correlated with a reduced amount of sugars in the leaves. Since the shade avoidance syndrome (SAS) is under control of phytochrome B (phyB) [4], the sucrose transporter StSUT4 is expected to be one of the phyB downstream signaling targets of this photoreceptor, and the expression of all three sucrose transporter genes of potato plants seem to be under control of the circadian clock [1].

In turn, in Arabidopsis, the involvement of miR156 and miR172 and their appropriate target genes in the induction of flowering under long day (LD) conditions is well investigated. Recent advances showed that the level of miR172 and miR156 which both are involved in regulating the transitions between developmental stages, i.e., from the juvenile to adult stage as well as in the transition from the vegetative to the generative phase (for a review, see [5]) seem to be sugar dependent in feeding experiments [6,7]. It has been shown that miR156 is repressed by glucose, as well as by sucrose. It is also shown in Arabidopsis that the age-dependent miR156 integrates sugar-dependent information about the nutritional status of the plant via the signaling molecule trehalose-6-phosphate with the age pathway for flower induction [8].

Only little is known about the role of miR172 and miR156 in Solanaceous plants. One flower inducing pathway via the florigenic protein flowering locus $\mathrm{T}$ (in the following called FT protein) involves the two microRNAs 156 and 172 and expression of FT is repressed by two AP2 transcription factors (SNZ and SMZ) which represent targets of miR172. Here, the induction of the florigenic FT gene occurs in a CONSTANS independent manner and seems to be mainly triggered via the sugar status in leaves. In potato, flowering and tuberization are triggered by the florigen and the tuberigen and both show similarities to flowering locus T from Arabidopsis. Potato plants possess as many as nine different FT homologues which are expressed in a photoperiod-dependent manner [9].

The aim of this study is the quantification of miR172 in the leaves of StSUT4-silenced and leaves of StSUT4-overexpressing plants in comparison to wild type plants. The question is whether sugar levels have an impact on the level of mature miR172 both in whole cut leaves as well as in whole in vitro plantlets that were supplemented with exogenous sugars. Our intention was to investigate whether similar mechanisms of sugar repression of miRNAs are valid for potato plants as previously observed in Arabidopsis.

\section{Results}

\subsection{StSUT4-RNAi Plants Are miR172 Over-Expressors}

We were interested in quantification of the level of miRNA172 in StSUT4-silenced plants since morphological observations revealed that the phenotype of StSUT4 silenced plants is very similar to what was observed for miR172 overexpressing plants [10] regarding internode elongation, early flowering, tuberization under non-inductive conditions, etc., [1]. Comparing source leaf RNA, we observed a significantly increased level of miR172 in one out of two independent transformant lines with reduced levels of StSUT4 expression (RNAi 2/5 and RNAi 2/16) compared to WT plants (Figure 1A,B). As a positive control, 35S::miR172 overexpressing potato plants have been used that show an even stronger upregulation of miR172 accumulation (Figure 1A). No significant increase in miR172 levels was observed in StSUT4-GFP overexpressing plants (Figure 1B).

Interestingly, the level of miR172 was significantly up-regulated in two independent sets of StSUT4 silenced plants: both StSUT4-RNAi plants from Solanum tuberosum ssp. tuberosum Désirée (Figure S1) as well as from Solanum tuberosum ssp. Andigena (Figure $1 \mathrm{~A}, \mathrm{~B})$, which is strongly photoperiod-dependent with respect to tuberization, showed significant up-regulation of miR172 accumulation in source leaves (Figure 1A,B). In sink leaves however, the level of miR172 is not increased in StSUT4-RNAi plants (RNAi 2/5 and RNAi 2/16), but in StSUT4-GFP overexpressing plants (StSUT4-GFP, Figure 1C). 
Further analysis of the level of miR172 in the shoot apical meristem of StSUT4-RNAi plants revealed increased levels of miRNA sink organs as well (Figure S2).

Detailed analysis of miR172 levels during the light period in potato wild type and StSUT4-silenced plants indicated a diurnal oscillation of this miRNA during the light period (Figure S3) when soluble sugar levels are increasing as well [1].

This might explain the similar phenotypic changes of miR172 over-expressors and StSUT4-RNAi plants $[1,10]$ and the question arose whether or not altered levels of soluble sugars in the leaves of StSUT4 silenced plants are responsible for this increase in the level of mature miR172 in those plants.

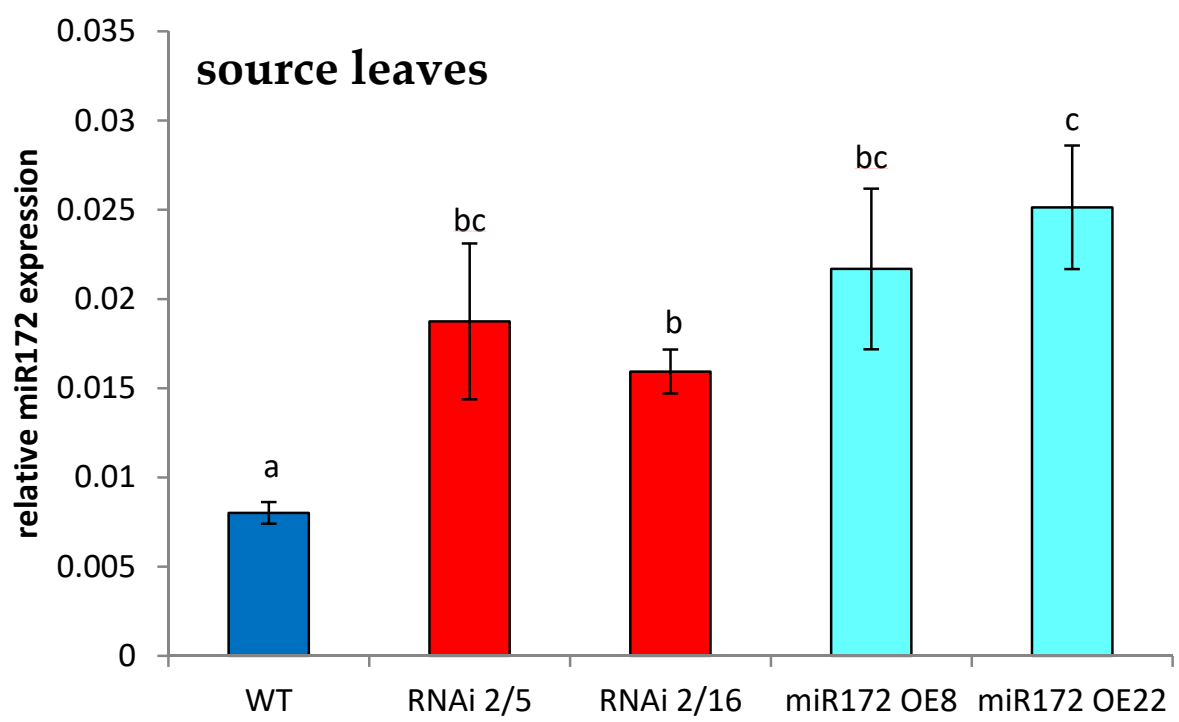

(A)

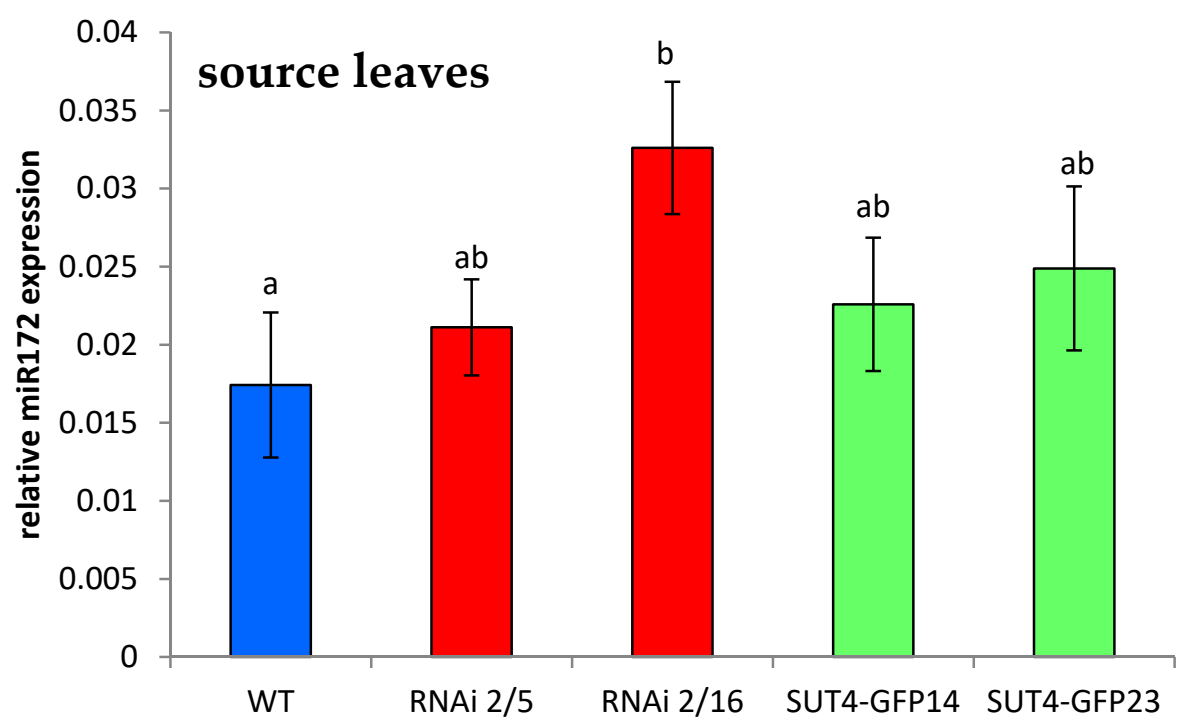

(B)

Figure 1. Cont. 


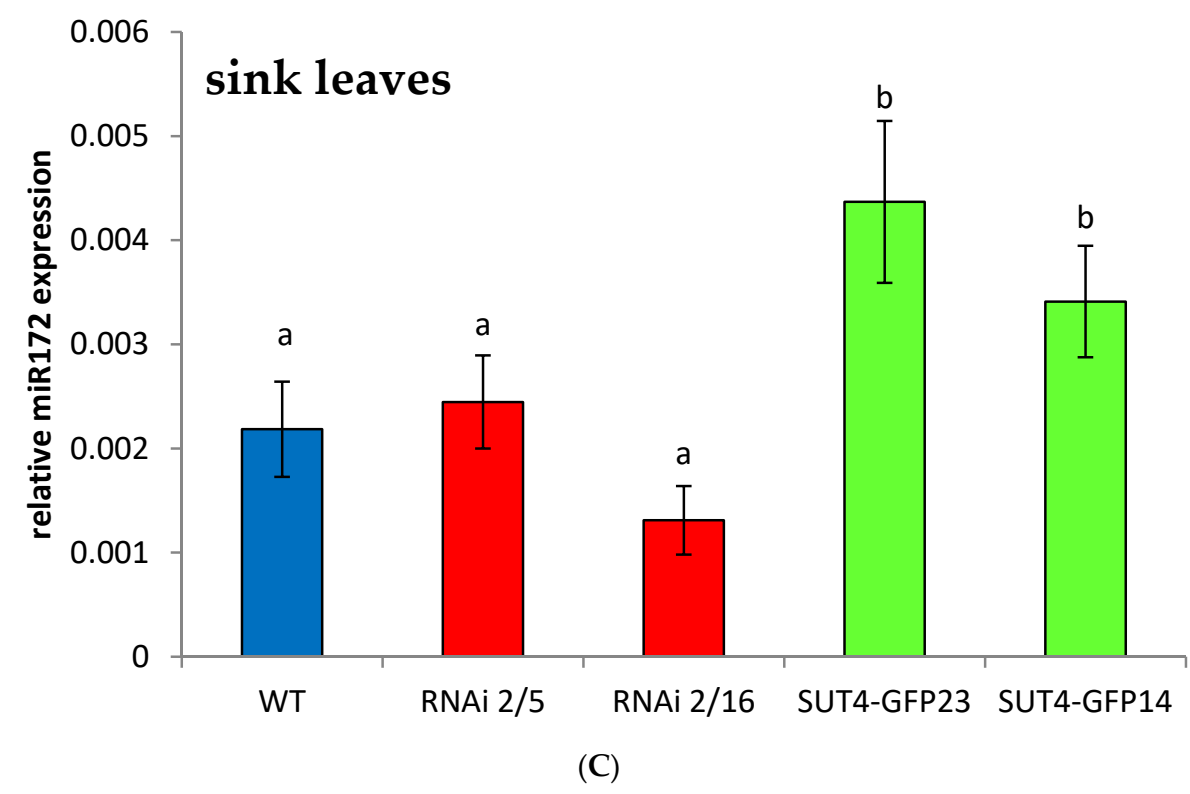

Figure 1. (A) Level of miR172 in source leaves of 35S::miR172 overexpressing potato plants (cyan bars) and StSUT4-RNAi plants (red bars) compared to Solanum tuberosum ssp. Andigena wild type plants (blue bar). 35S::miR172 overexpressing potato plants were previously shown to express increased amounts of miR172 [10] and were used as positive control. (B) Quantification of miR172 in source leaves of StSUT4-RNAi plants (red bars) and CaMV35S::StSUT4-GFP overexpressing plants (green bars). (C) Quantification of miR172 in sink leaves of StSUT4-RNAi plants (red bars) and CaMV35S::StSUT4-GFP overexpressing plants (green bars). Relative quantification was performed using 5SrRNA as a reference. StSUT4-RNAi plants were already described previously [1]. One way ANOVA was performed with $(\alpha=0.05)$. Significant differences are indicated by different letters.

\section{2. miR172 Is Induced by High Levels of Sucrose Both in Long Time and Short Time Experiments}

In order to test, whether the level of miR172 is affected by external sugar supply, we performed two different kinds of feeding experiment: one short time experiments using whole cut leaves from 6 weeks old adult plants grown under greenhouse conditions (Figure 2), or alternatively whole plantlets grown under sterile in vitro conditions in the phytochamber for 21 days on medium supplemented with different carbon sources (Figure 3).

The slight increase in miR172 transcript amounts during the short time experiment shown in Figure 2 when sugars are omitted from the medium or sorbitol is supplied might be explained by diurnal oscillation during the day. In the presence of $100 \mathrm{mM}$ of sucrose a significant increase in the level of miR172 is detectable after 2 and $3 \mathrm{~h}$ of incubation (Figure 2) suggesting that miR172 expression is sucrose-inducible. Glucose, as well, showed a transient increase in miR172 after $3 \mathrm{~h}$ of incubation (Figure S4).

Additionally, in plants cultured in vitro for longer time (Figure 3), the amount of miR172 is significantly higher if plants were grown on sucrose containing medium. In both cases, the amount of miR172 is more than doubled compared to the control treatment without any sugar. Whereas glucose application showed a transient increase in miR172 after $3 \mathrm{~h}$ of incubation in the short time experiment (Figure S4), no significant increase in the level of miR172 was observed in the long time experiment (Figure 3). 


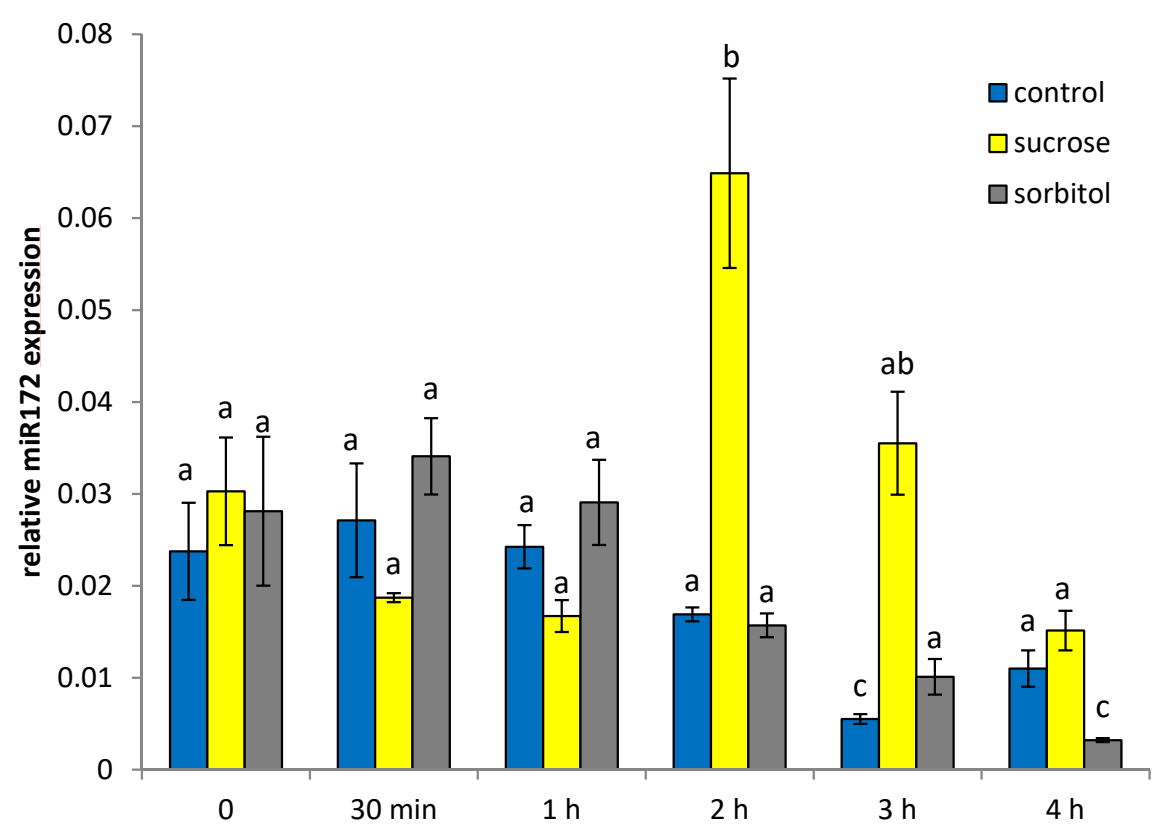

Figure 2. Quantification of miR172 in mature leaves depending on sugar supply in a short time experiment. Short time petiole experiment in the presence or absence of sugars was conducted with source leaves of 6 weeks old potato wild type plants (Solanum tuberosum variety Désirée). Sugars such as sucrose or sorbitol were supplied at a concentration of $100 \mathrm{mM}$ each in $2.5 \mathrm{mM}$ EDTA. Note that miR172 levels in the absence of sugars might oscillate diurnally during the day. Quantification was performed using 5SrRNA as a reference $(\alpha=0.05)$. Significant differences are indicated by different letters and SE is given.

\subsection{In Vitro Plants Grow Optimal in the Presence of Sucrose}

In order to test whether plant's performance depend on the carbon source, we propagate potato cuttings under sterile in vitro conditions on different sugars or without any additional sugar supply in the medium. After 21 days of growth on Murashige and Skoog (MS) Medium, the plantlets growing on $80 \mathrm{mM}$ sucrose containing medium behaved optimal with maximum plant height and maximum root length (Figure $3 \mathrm{~A}, \mathrm{~B}$ ), whereas plants grown on sorbitol containing medium or without any sugar supply remained much smaller.

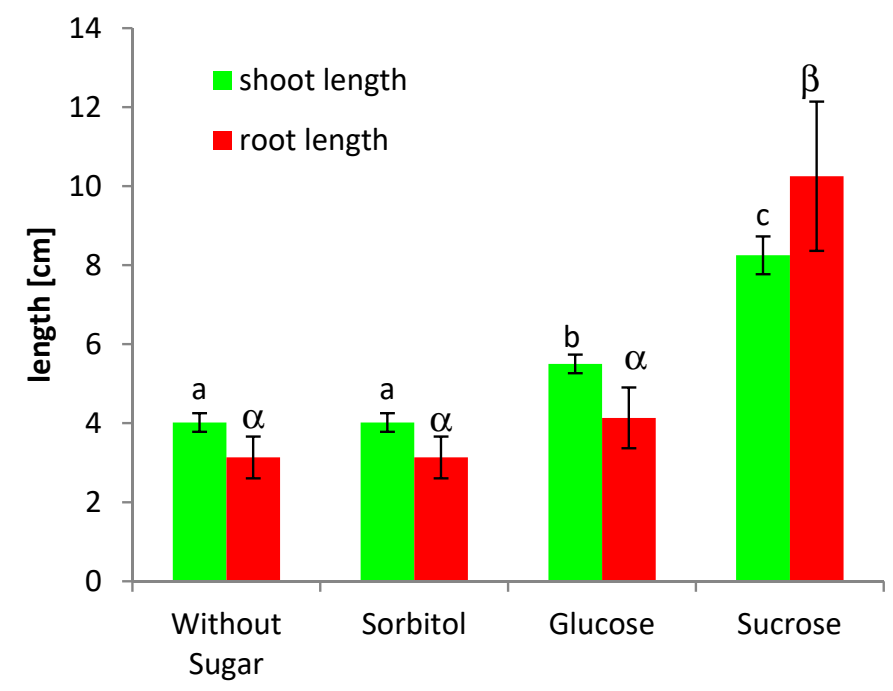

(A)

Figure 3. Cont. 


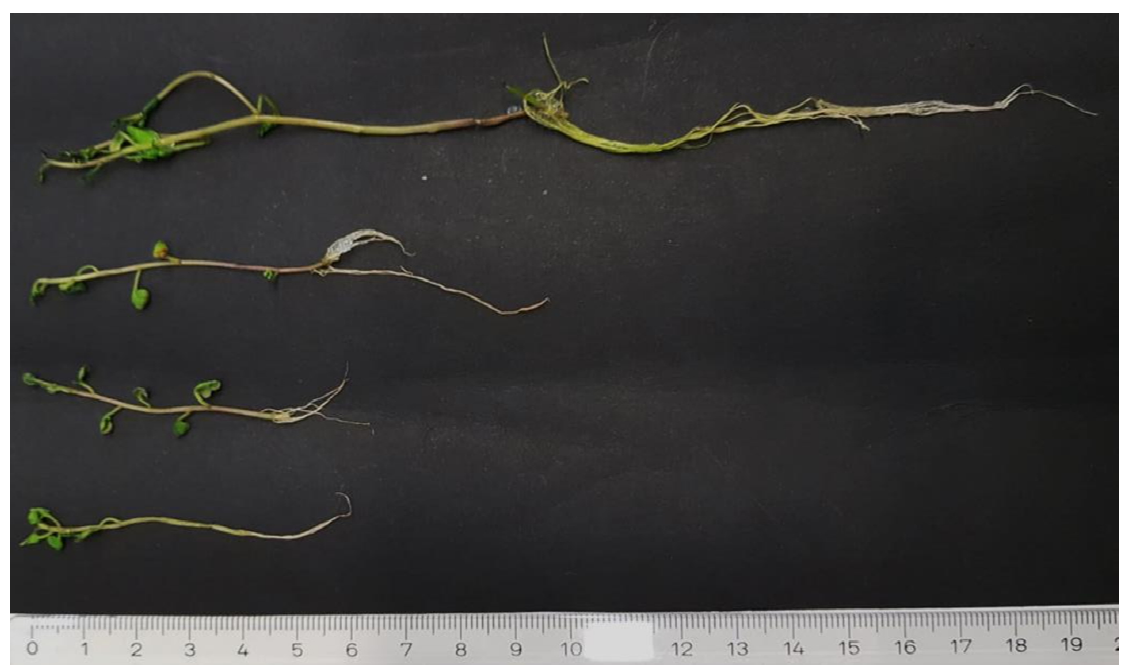

(B)

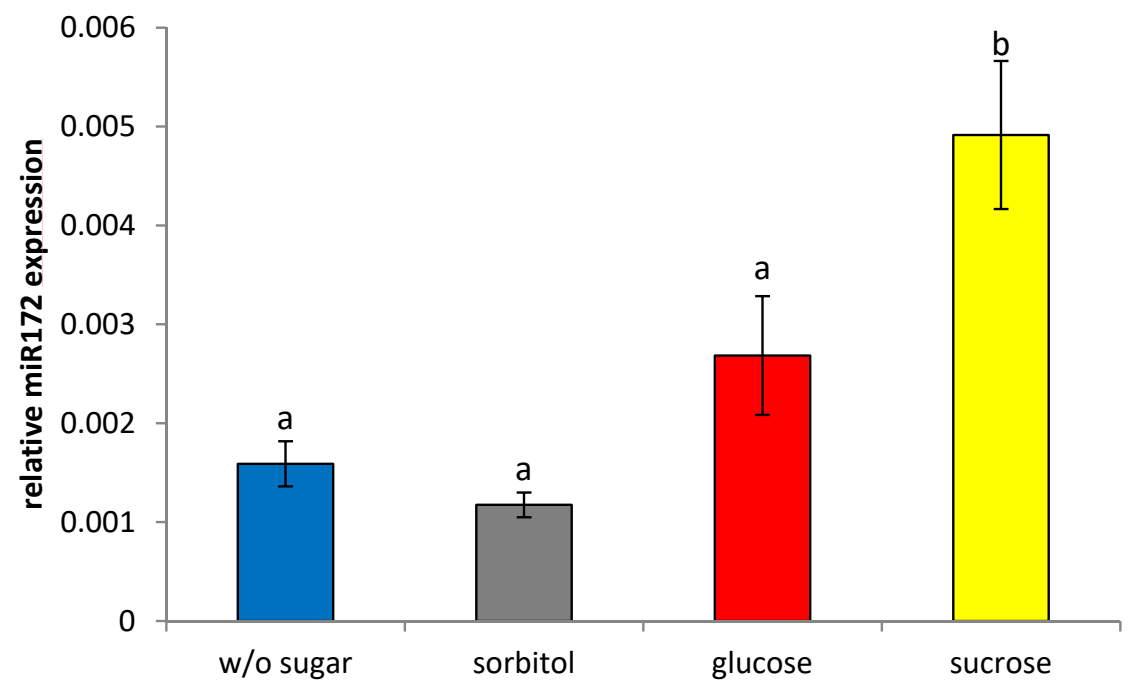

(C)

Figure 3. Growth and root morphology of potato wild type plants grown in vitro for 21 days on different carbon sources. Shoot and root length of six individual wild type potato plantlets were measured (A), as well as the total plant size (B) depending on the carbon source. Optimal growth and highest biomass production was observed when plantlets were grown on Murashige \& Skoog (MS) medium supplied with $80 \mathrm{mM}$ of sucrose. The standard error of the mean is given in (A) with $n=6$ individual wild type plants. (C) Quantification of miR172 in a long time experiments by qPCR using whole plantlets, which photos are shown in (A) after 21 days of growth under sterile conditions. Whereas the effect of glucose and sorbitol on the transcript amount of miR172 is statistically not significant, the accumulation of miR172 transcripts is significantly higher when sucrose has been added in a concentration of $80 \mathrm{mM}$. Quantification of miRNAs was performed with Solanum tuberosum ssp. tuberosum plants using 5SrRNA as a reference (with $p<0.001$ ). Significant differences are indicated by different letters.

\section{Discussion}

Potato plants overexpressing miR172 show graft-transmissible effects on flowering and tuberization, including promotion of flowering, acceleration of tuberization under moderately inductive photoperiods, induction of tuber formation under long days and shorter internode elongation [10]. Very similar phenomena were observed in StSUT4silenced potato plants [1]. Therefore, we were interested in the level of this miRNA is the transgenic StSUT4-silenced, as well as in StSUT4-overexpressing potato plants. Our results demonstrate increased levels of miR172 in StSUT4-silenced source leaves, whereas in sink 
leaves this miR172 increases when StSUT4 is overexpressed (Figure 1). This argues for StSUT4 acting upstream of miR172 in a sink-source dependent manner.

In sugar feeding experiments, an induction of miR172 in response to sucrose supply was observed in two kinds of experiments: direct sucrose feeding to cut petioles of adult leaves, as well as in a long term feeding experiments over a period of three weeks with in vitro grown plants (Figures 2 and 3). In both experiments, the miR172 expression was induced by sucrose application (Figures 2 and 3 ).

At the end of the light period, the efflux of sucrose was strongly enhanced in StSUT4inhibited potato plants [1]. In parallel, the level of soluble sugars that is decreased during the day, is also increased to high levels at the end of the light period ( $15 \mathrm{~h}$ Zeitgeber time, [1]). The miR172 quantification shown in Figure 1 would be consistent with increased levels of soluble sugars in source leaves as we measured at the end of the light period. The levels of soluble sugars and starch in sink leaves of StSUT4-silenced plants is rather increased [1] and sucrose peaks in the shoot apical meristem is shifted to earlier time points in the transgenic plants [1] which might be the reason for early flowering, and perhaps also for an earlier onset of tuberization in these plants.

At least for the soybean miR172, it is known that expression follows a diurnal rhythm [11] and it cannot be excluded that miR172 in potato oscillated as well diurnally. The slight increase in miR172 transcripts during the short time experiment shown in Figure 2 in samples without sugar or treated with sorbitol argues for such a diurnal change of the level of miR172 during the day (Supplementary Figure S3). Sucrose plays an important role in the entrainment of the circadian clock [12]. It cannot be excluded that changes in the level of miR172 are due to a shift in oscillation since the diurnal changes of soluble sugars is also shifted in StSUT4-silenced plants arguing for a disturbed entrainment of the circadian clock [1].

miR172 and miR156 are often engaged in identical regulatory pathways changing in opposite directions during plant development. In tomatoes, the overexpression of slymiR156a partially phenocopies the phenotype of $s f t$ mutants, with respect to late flowering, more abundant leaves, dwarfism, bushier structure, etc. The SINGLE FLOWER TRUSS (SFT) transcript amount is significantly down-regulated in 35S::miR156a overexpressing tomato plants suggesting that SFT is among the targets of miR156a. Comparing the effects of miR156 overexpression in Arabidopsis, rice, maize, and tomatoes, an evolutionary conserved function for miR156 was postulated [13].

In potato plants, miR156a was shown to be expressed mainly under long day (LD) conditions in leaves, whereas expression in stolons is higher under short day (SD) conditions [14]. miR156a is graft-transmissible and promoter analysis suggests a light-dependent expression [14]. In potato, miR156 seem to affect multiple growth traits as miR156 overexpression results in altered leaf and trichome morphology, aerial tuber production under SDs, delayed flowering, reduced stomatal density, reduced root biomass etc. It was excluded that miR156 acts as a repressor of tuberization and that miR156 function differs in LDs and in SDs [14]. In Arabidopsis, the level of miR156a and miR156c is negatively affected by sugar supply $[6,7,15]$. Further experiments are needed to answer the interplay between these two miRNAs in Solanaceous plants.

Regarding sucrose transporter StSUT4, it is assumed that StSUT4 acts as a pace-maker of circadian expression of sucrose transporters and sucrose efflux from leaves in a phyBdependent manner. Via time-dependent effects on the sugar level in source and sink tissues, StSUT4 thereby affects the level of the phloem-mobile miR172 and further downstream signaling events, potentially involving targeting of AP2-like transcription factors.

\section{Materials and Methods}

\subsection{Plant Material and Growth Conditions}

Solanum tuberosum L. subspecies andigena line 7540 was used as wild-type potato. Plants were propagated from single-node stem cuttings on Murashige and Skoog (MS) medium containing either $16 \mathrm{~g} \cdot \mathrm{L}^{-1}$ glucose, sucrose, sorbitol, or no sugar at all and $2 \mathrm{~g} \cdot \mathrm{L}^{-1}$ 
agar (Duchefa Biochemie, Haarlem, The Netherlands) at $23^{\circ} \mathrm{C}$ under LD conditions (16 h light and $8 \mathrm{~h}$ darkness). 35S:::miR172 overexpressing plants were kindly provided by Paula Suarez-Lopez [10]. In order to analyze the level of mature miR172 in StSUT4-RNAi plants were designed stem loop primers for qPCR according to [9]. As a positive control was used miR172 overexpressing potato plants that overexpress miR172 under control of the constitutive CaMV35S promoter [9]. The experiments were conducted on the transgenic plants in order quantification of the level of miRNAs. Plant material (source and sink leaves) for analyses were collected from 6 weeks old plants grown under long day (LD) conditions in the green house. The following experimental variants were used: wild type (WT), StSUT4-RNAi plants (RNAi2/5; RNAi2/16), CaMV35S::miR172-overexpressing plants (miR172OE8; miR172OE22), and StSUT4-overexpressing plants (SUT4-GFP23, SUT4GFP14). Yeast complementation experiments were performed in order to test whether C-terminal fusion of GFP to sucrose transporters affect sucrose transport capacity [16]. Sugar feeding experiments were performed with whole plantlets in long time experiments ( 3 weeks of growth with or without sugar) and whole cut leaves from 6 weeks old wild type potato plants in short time experiments ( 0 to $5 \mathrm{~h}$ incubation with or without sugar).

\subsection{Plant Material for Sugar Feeding Experiments}

Leaves of potato wild type plants grown under greenhouse conditions with a $16 \mathrm{~h} \mathrm{light-}$ $8 \mathrm{~h}$ dark cycle were harvested from 6 week old plants. Petioles of detached leaves were re-cut while submerged in water, $2.5 \mathrm{mM}$ EDTA was added to inhibit callose formation, and the cut petioles were transferred to the appropriate sugars solution containing $2.5 \mathrm{mM}$ EDTA in addition. Sugar concentration was $100 \mathrm{mM}$ glucose, sucrose, or sorbitol. The transcript amount of each miRNA was determined before the experiment $(\mathrm{t} 0)$ and samples were collected at the indicated period of time ( $30 \mathrm{~min}, 1 \mathrm{~h}, 2 \mathrm{~h}, 3 \mathrm{~h}$, and $4 \mathrm{~h}$ ). Experiments were conducted from 10 am till 2 pm under greenhouse conditions. Quantification by qPCR was conducted as indicated below.

\subsection{Analysis of miRNA Levels}

RNA was isolated from leaves or whole sterile plants. RNA extraction was performed using TRIzol (Invitrogen, Carlsbad, California, United States) according to the manufacturer's protocol. Reverse transcription was performed with the SuperScript Reverse Transcriptase (Thermo Fischer, Waltham, MA, USA) according to the manual. Reverse transcription was performed using oligo(dT) primers on approximately $1 \mu \mathrm{g}$ of total RNA after digestion with RNase-free DNase I (Fermentas/Thermo Scientific).

For the quantification of small RNAs, total RNA was reverse-transcribed using SuperScript Reverse Transcriptase (Thermo Fischer) following the manufacturer's protocol with $1 \mu \mathrm{L}$ of a 1:1 mixture of oligo-(dT) $)_{18}$ and the miRNA-specific stem-loop RT primers used in the priming step. The stem-loop RT primer and the oligonucleotide pair used for qRT-PCR analysis are shown in Table 1.

Table 1. Primers used for quantification of miRNAs.

\begin{tabular}{cr}
\hline miR156 stem loop primer & GTCGTATCCAGTGCAGGGTCCGAGGTATTCGCACTGGATACGACGTGCTC \\
\hline miR156 fw primer & GCGGCGGTGACAGAAGAGAGT \\
\hline miR172 stem loop primer & GTCGTATCCAGTGCAGGGTCCGAGGTATTCGCACTGGATACGACAGGGAT \\
\hline miR172 fw primer & CGGCGGTAGAATCTTGATGATG \\
\hline 5SrRNA stem loop primer & GTCGTATCCAGTGCAGGGTCCGAGGTATTCGCACTGGATACGACAGGGAT \\
\hline 5SrRNA fw primer & GGATGCGATCATACCAGCACT \\
\hline Universal rev primer & GTGCAGGGTCCGAGGT \\
\hline
\end{tabular}

Aliquots of $20 \mathrm{ng}$ of cDNA and primer (Table 1) concentration of $250 \mathrm{nM}$ were used for the subsequent qRT-PCR reaction. qRT-PCR was performed using ChamQ Universal 
$2 \times$ SYBR $^{\circledR}$ Green Mix (Vazyme Biotech, Nanjing, China) reaction mixture in a Bio-Rad CFX System (Bio-Rad Laboratories, Feldkirchen, Germany) in $10 \mu \mathrm{L}$ reaction volumes. Experiments were performed in biological triplicates.

The qRT-PCR reactions were performed in the following cycling conditions: activation for 10 min at $95^{\circ} \mathrm{C}$, denaturation at $95^{\circ} \mathrm{C}$ for $15 \mathrm{~s}$, annealing for $15 \mathrm{~s}$ at $60^{\circ} \mathrm{C}$ and elongation for $15 \mathrm{sec}$ at $72{ }^{\circ} \mathrm{C}$, in a program of 45 cycles. Relative quantification of transcript amounts was always calculated in relation to the respective transcript level of appropriate reference genes (5S rRNA) and given as relative expression $\left(2^{(-\Delta \Delta C t)}\right)$.

For normalization, $5 \mathrm{~S}$ ribosomal RNA was reverse transcribed by stem-loop primer $5 \mathrm{~S}$ rRNA stem loop primer and amplified by 5SrRNAfw and universal rev in the same conditions as for miR172 and miR156. PCR specificity was checked by melting curve analysis, and data were analyzed using the $2^{-\Delta \Delta C t}$ method [17].

\subsection{Primers for qPCR Analysis \\ 4.5. Statistical Analysis}

Statistical evaluation of real time data was performed with $n$ between 6 and 12 individuals (three biological replicates and two technical replicates). To test whether phenotypical traits differ between treatments or whether the individuals of single transgenic lines differ significantly in their expression level from WT plants, factorial analysis of variance (ANOVA) was carried out in accordance with the experimental design with $\alpha=0.05$ using the SigmaPlot or Excel software. Significant differences are indicated by different letters and the SE is given.

\section{Conclusions}

The results of our study demonstrate considerably higher level of miR172 in several transformant lines with reduced levels of StSUT4 expression compared to wild type plants. Moreover, sucrose had a positive impact on the accumulation of miR172 both in leaves of whole plantlets in long time experiments as well as in whole cut leaves in short time experiments. In fact, potato and Arabidopsis share similar signal transduction components regarding the flower inducing signaling pathway and miRNAs seem to play an important role in both pathways $[18,19]$. Sugar dependent effects on the expression level of the age-dependent miR156 are already described in case of Arabidopsis which with respect to flowering control is assumed to represent a long day plant, whereas potato with respect to tuberization is short day-dependent. Here, the level of miR172 which targets members of the AP2 transcription factors seems to be sugar-inducible as shown in short time and long-time experiments. The sugar status in leaves of potato, which is an important crop, has severe impact on downstream signaling events affecting flowering and tuberization. It is obvious that miR172 as well as sucrose transporter StSUT4 are involved in those pathways.

Supplementary Materials: The following are available online at https:/ / www.mdpi.com/1422-006 $7 / 22 / 3 / 1455 / s 1$.

Author Contributions: Conceptualization, C.K. and V.G.; methodology, C.K.; experiments, V.G. and A.H.; validation, C.K., V.G., and A.H.; writing-original draft preparation, C.K.; writing-review and editing, V.G.; visualization, C.K. and V.G.; supervision, C.K. All authors have read and agreed to the published version of the manuscript.

Funding: This research was funded by the German research foundation (DFG) SPP1530 to C.K. and the woman's advancement panel (KFF) of the Institute of Biology, Humboldt-Universtiät zu Berlin to V.G.

Institutional Review Board Statement: Not applicable.

Informed Consent Statement: Not applicable.

Data Availability Statement: The data that support the findings of this study are available from the corresponding author upon reasonable request. 
Acknowledgments: We acknowledge care of greenhouse plants by Angelika Pötter and helpful discussion with Salomé Prat. We gratefully acknowledge financial support by the woman's advancements panel (KFF) of the Institute of Biology, Humboldt-Universität who is covering the publication fees.

Conflicts of Interest: The authors declare no conflicts of interest. The funders had no role in the design of the study; in the collection, analyses, or interpretation of data; in the writing of the manuscript, or in the decision to publish the results.

$\begin{array}{ll}\text { Abbreviations } \\ \text { EDTA } & \text { Ethylenediaminetetraacetic acid } \\ \text { GFP } & \text { Green fluorescent protein } \\ \text { miRNA } & \text { Micro RNA } \\ \text { LD } & \text { Long day } \\ \text { RNA } & \text { Ribonucleic acid } \\ \text { SD } & \text { Short day } \\ \text { SUT } & \text { Sucrose transporter } \\ \text { RT-PCR } & \text { Reverse transcription-polymerase chain reaction }\end{array}$

\section{References}

1. Chincinska, I.A.; Liesche, J.; Krügel, U.; Michalska, J.; Geigenberger, P.; Grimm, B.; Kühn, C. Sucrose transporter StSUT4 from potato affects flowering, tuberization, and shade avoidance response. Plant Physiol. 2008, 146, 515-528. [CrossRef]

2. Harmer, S.L.; Kay, S.A. Positive and negative factors confer phase-specific circadian regulation of transcription in Arabidopsis. Plant Cell 2005, 17, 1926-1940. [CrossRef]

3. Chincinska, I.; Gier, K.; Krügel, U.; Liesche, J.; He, H.; Grimm, B.; Harren, F.J.; Cristescu, S.M.; Kühn, C. Photoperiodic regulation of the sucrose transporter StSUT4 affects the expression of circadian-regulated genes and ethylene production. Front. Plant Sci. 2013, 4, 26. [CrossRef]

4. Smith, H.; Whitelam, G.C. The shade avoidance syndrome: Multiple responses mediated by multiple phytochromes. Plant Cell Environ. 1997, 20, 840-844. [CrossRef]

5. Fornara, F.; Coupland, G. Plant phase transitions make a SPLash. Cell 2009, 138, 625-627. [CrossRef] [PubMed]

6. Yang, L.; Xu, M.; Koo, Y.; He, J.; Poethig, R.S. Sugar promotes vegetative phase change in Arabidopsis thaliana by repressing the expression of MIR156A and MIR156C. eLife 2013, 2, e00260. [CrossRef] [PubMed]

7. Yu, S.; Cao, L.; Zhou, C.M.; Zhang, T.Q.; Lian, H.; Sun, Y.; Wu, J.; Huang, J.; Wang, G.; Wang, J.W. Sugar is an endogenous cue for juvenile-to-adult phase transition in plants. eLife 2013, 2, e00269. [CrossRef]

8. Wahl, V.; Ponnu, J.; Schlereth, A.; Arrivault, S.; Langenecker, T.; Franke, A.; Feil, R.; Lunn, J.E.; Stitt, M.; Schmid, M. Regulation of flowering by trehalose-6-phosphate signaling in Arabidopsis thaliana. Science 2013, 339, 704-707. [CrossRef] [PubMed]

9. Navarro, C.; Cruz-Oro, E.; Prat, S. Conserved function of FLOWERING LOCUS T (FT) homologues as signals for storage organ differentiation. Curr. Opin. Plant Biol. 2015, 23, 45-53. [CrossRef] [PubMed]

10. Martin, A.; Adam, H.; Diaz-Mendoza, M.; Zurczak, M.; Gonzalez-Schain, N.D.; Suarez-Lopez, P. Graft-transmissible induction of potato tuberization by the microRNA miR172. Development 2009, 136, 2873-2881. [CrossRef] [PubMed]

11. Wang, T.; Sun, M.Y.; Wang, X.S.; Li, W.B.; Li, Y.G. Over-Expression of GmGla-Regulated Soybean miR172a Confers Early Flowering in Transgenic Arabidopsis thaliana. Int. J. Mol. Sci. 2016, 17, 645. [CrossRef] [PubMed]

12. Haydon, M.J.; Mielczarek, O.; Robertson, F.C.; Hubbard, K.E.; Webb, A.A. Photosynthetic entrainment of the Arabidopsis thaliana circadian clock. Nature 2013, 502, 689-692. [CrossRef] [PubMed]

13. Zhang, X.; Zou, Z.; Zhang, J.; Zhang, Y.; Han, Q.; Hu, T.; Xu, X.; Liu, H.; Li, H.; Ye, Z. Over-expression of sly-miR156a in tomato results in multiple vegetative and reproductive trait alterations and partial phenocopy of the sft mutant. FEBS Lett. 2011, 585, 435-439. [CrossRef] [PubMed]

14. Bhogale, S.; Mahajan, A.S.; Natarajan, B.; Rajabhoj, M.; Thulasiram, H.V.; Banerjee, A.K. MicroRNA156: A potential grafttransmissible microRNA that modulates plant architecture and tuberization in Solanum tuberosum ssp. andigena. Plant Physiol. 2014, 164, 1011-1027. [CrossRef] [PubMed]

15. Ponnu, J.; Schlereth, A.; Zacharaki, V.; Dzialo, M.A.; Abel, C.; Feil, R.; Schmid, M.; Wahl, V. The trehalose 6-phosphate pathway impacts vegetative phase change in Arabidopsis thaliana. Plant J. 2020, 104, 768-780. [CrossRef] [PubMed]

16. Lalonde, S.; Weise, A.; Walsh, R.P.; Ward, J.M.; Frommer, W.B. Fusion to GFP blocks intercellular trafficking of the sucrose transporter SUT1 leading to accumulation in companion cells. BMC Plant Biol. 2003, 3, 8. [CrossRef] [PubMed]

17. Livak, K.J.; Schmittgen, T.D. Analysis of relative gene expression data using real-time quantitative PCR and the 2(-Delta Delta C(T)) Method. Methods 2001, 25, 402-408. [CrossRef] [PubMed] 
18. Kondhare, K.R.; Natarajan, B.; Banerjee, A.K. Molecular signals that govern tuber development in potato. Int. J. Dev. Biol. 2020, 64, 133-140. [CrossRef] [PubMed]

19. Natarajan, B.; Bhogale, S.; Banerjee, A.K. The essential role of microRNAs in potato tuber development: A mini review. Ind. J. Plant Physiol. 2017, 22, 401-410. [CrossRef] 